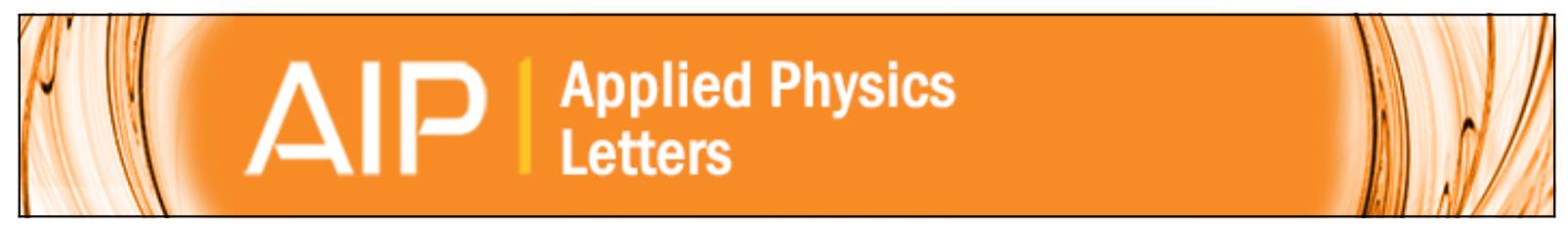

Observation of enhanced defect emission and excitonic quenching from spherically indented $\mathrm{ZnO}$

V. A. Coleman, J. E. Bradby, C. Jagadish, and M. R. Phillips

Citation: Applied Physics Letters 89, 082102 (2006); doi: 10.1063/1.2338552

View online: http://dx.doi.org/10.1063/1.2338552

View Table of Contents: http://scitation.aip.org/content/aip/journal/apl/89/8?ver=pdfcov

Published by the AIP Publishing

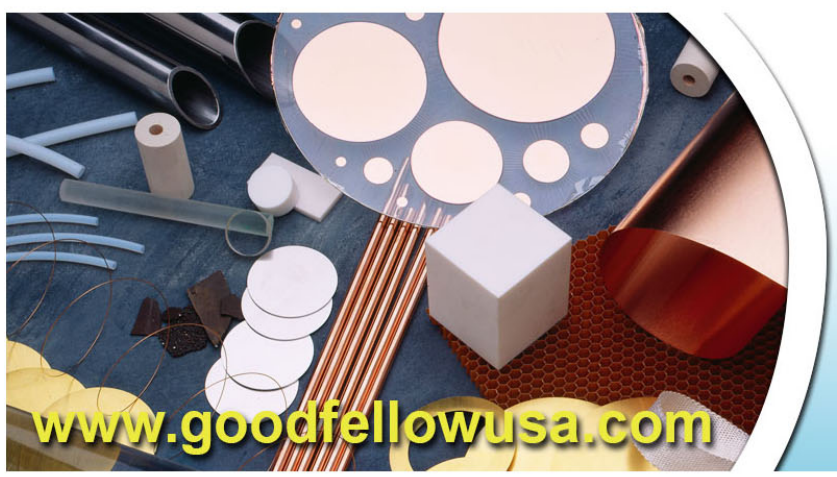

BOOdFEl|OII metals • ceramics • polymers composites • compounds • glasses

Save $5 \%$ • Buy online 70,000 products $\cdot$ Fast shipping 


\title{
Observation of enhanced defect emission and excitonic quenching from spherically indented $\mathrm{ZnO}$
}

\author{
V. A. Coleman, ${ }^{\text {a) }}$ J. E. Bradby, and C. Jagadish \\ Department of Electronic Materials Engineering, Research School of Physical Sciences and Engineering, \\ The Australian National University, Canberra, Australian Capital Territory 0200, Australia \\ M. R. Phillips \\ Microstructural Analysis Unit, The University of Technology Sydney, Broadway, New South Wales 2007, \\ Australia
}

(Received 9 February 2006; accepted 17 July 2006; published online 23 August 2006)

\begin{abstract}
The influence of spherical nanoindentation on the band edge and deep level emission of single crystal $c$-axis $\mathrm{ZnO}$ has been studied by cathodoluminescence (CL) spectroscopy and monochromatic imaging. Excitonic emission is quenched at the indent site and defect emission in the range of $450-720 \mathrm{~nm}$ is enhanced. Analysis of CL monochromatic images and spectra suggests that at least two different defect states are responsible for the broad defect emission band. Additionally, the indents result in a strong crystallographic dependence of the defect emission, producing a rosette feature with $[11 \overline{2} 0][2 \overline{1} \overline{1} 0]$, and $[1 \overline{2} 10]$ orientations that reflect the star-shaped luminescence quenching observed at the excitonic peak $(390 \mathrm{~nm})$. (C) 2006 American Institute of Physics.
\end{abstract} [DOI: $10.1063 / 1.2338552]$

With a wide band gap of $\sim 3.4 \mathrm{eV}$ and a large exciton binding energy of $60 \mathrm{meV}$ at $300 \mathrm{~K}$, it is anticipated that $\mathrm{ZnO}$ should produce room temperature devices operating with high efficiency and low power thresholds. "When combined with other attractive properties, including radiation hardness, a wide chemistry offering many possibilities for wet chemical etching, commercial availability of high quality single crystal substrates, opportunities for band gap engineering by alloying with $\mathrm{MgO}$ and $\mathrm{CdO}$, and proven technologies for the growth of epitaxial layers, $\mathrm{ZnO}$ strongly rivals $\mathrm{GaN}$ as a viable material for the production of blue and ultraviolet light emitting and laser diodes. ${ }^{2-6}$ However, the defect structure of $\mathrm{ZnO}$ and the origin of the visible defect band associated with this material are still the subject of controversy. Given the current difficulty in achieving $p$-type $\mathrm{ZnO}$, a thorough understanding of the nature of defects in this material is highly desirable. It is widely accepted that defect luminescence falls into three main bands: a green luminescence (GL) band around $510 \mathrm{~nm}$, a yellow luminescence (YL) band around $570 \mathrm{~nm}$, and a red/orange luminescence (RL) band at $\sim 650 \mathrm{~nm}^{7}$

In this letter, the optically active defect structure of $\mathrm{ZnO}$ is studied by combining nanoindentation with cathodoluminescence (CL) spectroscopy and monochromatic imaging. A number of CL studies have previously been conducted on bulk $c$-axis oriented $\mathrm{ZnO}$, concentrating on the quenching of the band edge emission to produce [11 $\overline{2} 0],[2 \overline{1} \overline{1} 0]$, and $[1 \overline{2} 10]$ oriented rosette features, and at higher loads, star features which are offset to the rosettes by $\sim 30^{\circ}$ along $[0 \overline{1} 10],[1 \overline{1} 00]$, and [1010] directions. ${ }^{8-10}$ Similar rosettes have also been observed by scanning Kelvin probe microscopy measurements of indents. ${ }^{11}$ Although these publications indicate that the rosettes arise as a result of dislocations and defects in the material, none of them attempt to further probe the defect structure of the material. Takkouk et al. ${ }^{10}$ provide

\footnotetext{
${ }^{a)}$ Electronic mail: victoria.coleman@anu.edu.au
}

an in depth analysis of the crystallographic dependence of the excitonic luminescence quenching in this materials system and suggest that indentation gives rise to two types of dislocation defects in $\mathrm{ZnO}$ : one $\mathrm{Zn}$-atom related and one $\mathrm{O}$-atom related. They do not, however, record any increase in visible luminescence following indentation. This study aims to extend the existing indentation studies further by looking at the deep level emission of nanoindented $\mathrm{ZnO}$ with $\mathrm{CL}$ spectroscopy and imaging and to provide both spatial and emission-energy information about the nature of these highly controversial defects.

The experiments were conducted on the oxygen face of $c$-axis bulk $n$-type $\mathrm{ZnO}$ purchased from Cermet, Inc. These are high quality single crystals with a nominal carrier concentration of $\sim 1 \times 10^{17} \mathrm{~cm}^{-3}$ and an estimated dislocation density of $\sim 1 \times 10^{4} \mathrm{~cm}^{-2}$. Aside from chemical degreasing and cleaning, no further changes were made to the asreceived surface. Following cleaning, the sample was cut into four pieces. Each piece was then indented in an ultramicro indentation system 2000 at room temperature using a spherical indenter of radius $\sim 4.3 \mu \mathrm{m}$. An array of 15 indents with maximum loads of 100 and $200 \mathrm{mN}$ was made on the surface of each sample. The indents were spaced such that the damage caused by one indent would not affect the surrounding indents. From previous experience with this material system, it was not expected that these loads would induce any cracking or phase transformations in the $\mathrm{ZnO}$.,12

After indentation, one sample was reserved as a reference while the remaining three samples were rapid thermally annealed at 400,600 , and $800{ }^{\circ} \mathrm{C}$, respectively, for $60 \mathrm{~s}$ under an Ar ambient. This step was implemented to monitor the recovery of the indented regions with annealing temperature. The samples were then loaded into JEOL35C scanning electron microscope (SEM) fitted with an Oxford Instruments MonoCL2 system for CL spectroscopy and monochromatic imaging. The CL spectra and images were measured using a Hamamatsu R943-02 Peltier cooled photomultiplier tube and a 1200 lines/mm grating blazed at $500 \mathrm{~nm}$. The accelerating 


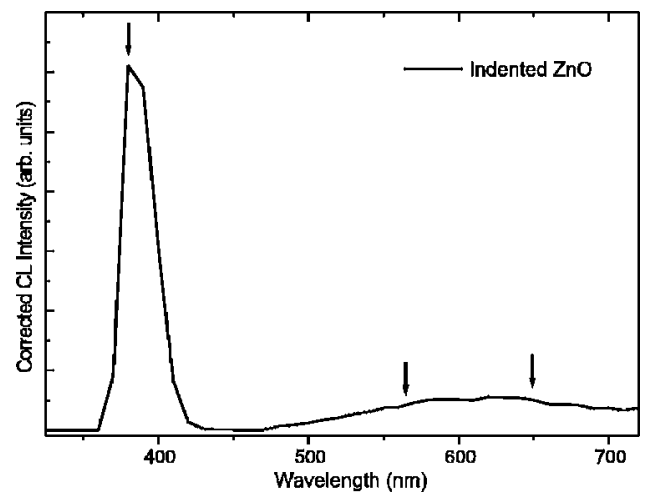

FIG. 1. Corrected $\mathrm{CL}$ spectra taken from the indented $\mathrm{ZnO}$ annealed at $600{ }^{\circ} \mathrm{C}$. The deep level emission has been enhanced for clarity. Arrows highlight the three wavelengths $(390,560$, and $650 \mathrm{~nm})$ used for CL monochromatic imaging.

voltage was $25 \mathrm{kV}$ and all measurements were made at $77 \mathrm{~K}$. All spectra were corrected for the system response of the detector.

In Fig. 1, a typical $\mathrm{CL}$ spectrum of indented $\mathrm{ZnO}$ is shown. In this case, the $600{ }^{\circ} \mathrm{C}$ annealed sample has been chosen since it shows the highest intensity of deep level emissions. The arrows on the CL spectrum correspond to the three wavelengths $(390,560$, and $650 \mathrm{~nm})$ chosen for monochromatic CL imaging. It should be noted that the GL band in these samples is very weak, with the deep level emissions being dominated by the YL and RL bands. The GL may, however, contribute in part to some of the YL intensity (assuming it has a broad Gaussian structure).

Figure 2 shows a $4 \times 4$ array of SEM (top row) and CL images. These images were all taken under the same microscope and monochromator conditions with the same integration time (10 s) to ensure that the images could be accurately compared. The image taken at the excitonic line $(390 \mathrm{~nm})$ shows quenching of the luminescence around the indent site in a characteristic six-pointed star formation. ${ }^{9,10}$ As previously observed, ${ }^{8-11}$ between the arms of the star there are quenching lines extending radially out from the center of the indent site in the $[11 \overline{2} 0][\overline{1} \overline{1} 0]$, and $[1 \overline{2} 10]$ directions, form-

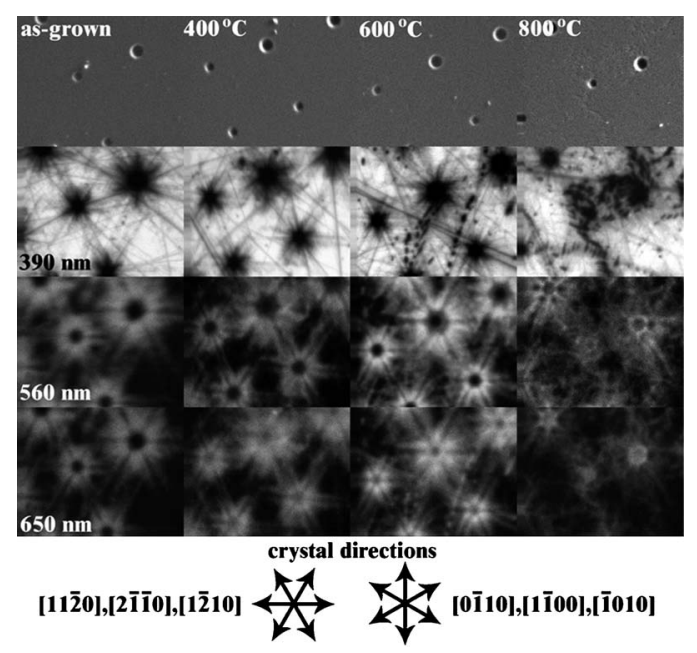

FIG. 2. SEM (top row) and CL monochromatic imaging of the four indented samples taken at the three wavelengths highlighted in Fig. 1. The imaging conditions and magnification were kept constant for all images, as was the image integration/acquisition time. The horizontal field width is $168 \mu \mathrm{m}$, and the indents shown are for 100 and $200 \mathrm{mN}$ loads. The main crystallographic directions are indicated for clarity. ing a rosette. Other sources of quenching in the $390 \mathrm{~nm}$ images arise due to sample polishing damage and minor surface scratching. The excitonic quenching is evident over all wavelengths, although at $800{ }^{\circ} \mathrm{C}$, it appears that there has been some recovery of the luminescence in the arms of the stars.

At $560 \mathrm{~nm}$, there is clear luminescence around the indent site. With annealing, this luminescence becomes increasingly structured and a well defined hexagon becomes evident around the indent, surrounded by a ring of defect luminescence. The defect luminescence follows the same rosette pattern as for the band edge emission; however, unlike the band edge emission in which there are double lines extending radially from the indent region, the quenching occurs as a single line extending from the points of the hexagon within the indent site. The lines are offset by $30^{\circ}$ with respect to the quenched arms of the star formation seen at $390 \mathrm{~nm}$ and correspond to the $[0 \overline{1} 10][1 \overline{1} 00]$, and $[\overline{1010}]$ directions. ${ }^{10}$ These lines might be indicative of nonradiative defect bands caused by the defects extending away from the indent site or by strain-induced migration away from the defect bands. This is not surprising as indentation results in the creation of large strain fields around the indent, with indentationinduced defects traveling far beyond the indent site in $\mathrm{ZnO}{ }^{12}$ Around these quenched lines, however, the $560 \mathrm{~nm}$ luminescence has greatest intensity suggesting that these defects act as clustering sites for the optically active point defects. The fact that the structure of the luminescence improves with annealing further supports this, with the energy available during annealing enhancing the mobility of point defects and allowing them to cluster to these high-strain regions. At $800{ }^{\circ} \mathrm{C}$ the luminescence intensity has dropped significantly, suggesting that the point defects are being annealed out at higher temperatures. It is anticipated that with a longer annealing time, most of the point defects would be annihilated at $800{ }^{\circ} \mathrm{C}$, as consistent with other annealing studies of the stability of defects in $\mathrm{ZnO}{ }^{13}$

In the case of the $650 \mathrm{~nm}$ images, the as-grown image looks almost identical to that of the image taken at $560 \mathrm{~nm}$. However, with annealing, the luminescence patterns start changing subtly. At $650 \mathrm{~nm}$ the luminescence seems to migrate more into the center of the indent site. This is most evident in the $600{ }^{\circ} \mathrm{C}$ annealed sample, where the luminescence originates from much closer to the indent site than for the same sample imaged at $560 \mathrm{~nm}$. Also, in this image a star shape of bright luminescence offset by $30^{\circ}$ with respect to

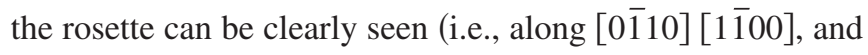
[1010] directions). This image seems to correlate much more strongly with that of the quenched areas of the band edge emission. At $800{ }^{\circ} \mathrm{C}$, the luminescence intensity is again dramatically reduced suggesting defect recovery, although the increased intensity from the indent site is still evident. These changes in the spatial positioning of the luminescence are indicative of two things. Firstly is that the deep level band of $\mathrm{ZnO}$ is made up of at least two distinct defects, and secondly is that the mobility of these defects within the $\mathrm{ZnO}$ is quite different as evidenced by the spatial redistribution of the luminescence around the indent site following annealing. The observation of at least two distinct defect states is consistent with other work.

Turning now to a spectral analysis of the deep level emissions surrounding the indent, from Fig. 3(a) it can be seen that the luminescence reaches maximum intensity after 


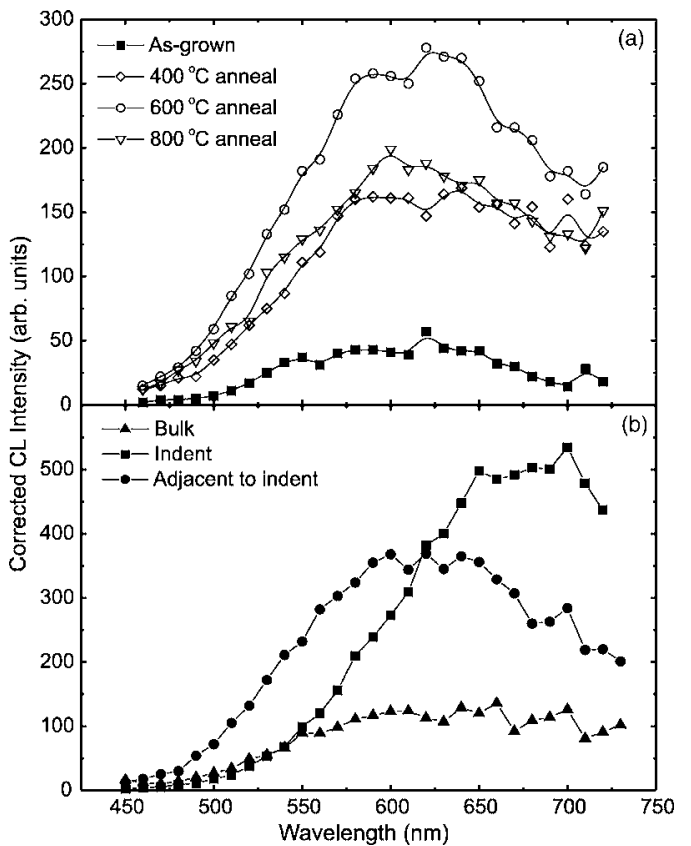

FIG. 3. (a) CL spectra of the deep level emissions of the four indented $\mathrm{ZnO}$ samples. (b) CL spectra taken from the $600{ }^{\circ} \mathrm{C}$ annealed and indented sample at different positions around the indent. "Indent" corresponds to spectra taken from the indent region only. "Adjacent to indent" corresponds to spectra taken directly to the side of the indent within the rosetteluminescence region. "Bulk" corresponds to the deep level luminescence seen from $\mathrm{ZnO}$ away from the indent site and damaged region. All spectra were taken with the same conditions, and (b) was taken with a horizontal field width of $89 \mu \mathrm{m}$.

being annealed at $600{ }^{\circ} \mathrm{C}$ for $60 \mathrm{~s}$. This intensity increase could be firstly the result of the indent acting as a clustering site for point defects already present in the material, undergoing maximum diffusion at this temperature. It could also be the result of point defects formed during the annealing process itself. In Fig. 3(b), the spectra taken from areas around the indent site are shown. In this case, the spectra are from the sample annealed at $600{ }^{\circ} \mathrm{C}$, since this shows the maximum defect intensity. By measuring the deep level emissions far away from the indent in the bulk of the sample (solid triangles), it would appear from the shape of the curve that the defect(s) responsible for the $560 \mathrm{~nm}$ emission is (are) most prevalent. We will assume that this luminescence is dominated by the YL band, with only a small contribution from the GL band. The spectrum taken directly adjacent to the indent (solid black circles) looks similar in shape to that of the bulk, with the intensity again being maximum at around $560 \mathrm{~nm}$. The spectrum recorded directly over the indent site, however, (shown here as the solid black squares) has a quite different shape. While it obviously contains a component of luminescence at $560 \mathrm{~nm}$, there is a significant shift in the peak intensity to $\sim 675 \mathrm{~nm}$. At $650 \mathrm{~nm}$ therefore we are mostly probing the RL. This is further evidence of the existence of at least two distinct optically active point defect states within the sample.

The fact that the spectrum taken adjacent to the indent site matches that of the bulk quite closely suggests that luminescence from this region is dominated by an existing and energetically favorable defect within the material that clusters to this site during annealing. $\mathrm{Zn}$ vacancies are known to have the lowest defect formation energy in $\mathrm{ZnO},{ }^{14}$ and thus a defect state involving $\mathrm{Zn}$ vacancies may be responsible for the deep emissions around $560 \mathrm{~nm}(\mathrm{YL})$; however, emission at this wavelength has also been previously attributed to lithium impurities in $\mathrm{ZnO}^{7} \mathrm{GL}$, which does not appear to play a significant role in these samples, is usually attributed to oxygen vacancies. ${ }^{15,16}$

The defect state responsible for the RL within the indent site may arise more from point defects introduced during indentation itself and be less energetically favorable for formation during $\mathrm{ZnO}$ growth. This suggests that the RL may also be related to an intrinsic defect complex in $\mathrm{ZnO}$ as opposed to an impurity.

Finally, it is useful to note that the relatively low CL intensity measured in the bulk is much less than the intensity seen for this sample when including the indents. This suggests that the enhancement of the deep level luminescence around the indent site at this temperature is more likely to be a result of clustering of existing point defects than the formation of new defects due to heating.

In conclusion, CL spectroscopy and monochromatic imaging have been used to study defects introduced into $\mathrm{ZnO}$ single crystals during nanoindentation. The data suggest that nanoindentation sites act as clustering sites for defects, and at least two defect states make up the deep level emissions. These defect states are expected to be intrinsic point defects that are both created during indentation and attracted to the highly strained indent site during annealing. We tentatively assign the YL to a zinc-vacancy related transition. It is hoped that the results of this study will lead to further work on the identification of the point defects responsible for the deep level emissions seen in $\mathrm{ZnO}$.

The authors wish to acknowledge the Australian Research Council for its financial support.

${ }^{1}$ D. C. Look, R. L. Jones, J. R. Sizelove, N. Y. Garces, N. C. Giles, and L. E. Haliburton, Phys. Status Solidi A 195, 171 (2003).

${ }^{2}$ C. Coskun, D. C. Look, G. C. Farlow, and J. R. Sizelove, Semicond. Sci. Technol. 19, 752 (2004).

${ }^{3}$ S. J. Pearton, D. P. Norton, K. Ip, Y. W. Heo, and T. Steiner, Prog. Mater. Sci. 50, 293 (2005).

${ }^{4}$ D. C. Look, Mater. Sci. Eng., B 80, 383 (2001).

${ }^{5}$ V. A. Coleman, H. H. Tan, C. Jagadish, S. O. Kucheyev, and J. Zou, Appl. Phys. Lett. 87, 231912 (2005).

${ }^{6}$ V. A. Coleman, M. Buda, H. H. Tan, C. Jagadish, M. R. Phillips, K. Koike, S. Sasa, M. Inoue, and M. Yano, Semicond. Sci. Technol. 21, L25 (2006).

${ }^{7}$ U. Özgür, Y. I. Alivov, C. Liu, A. Teke, M. A. Reshchikov, S. Doŭan, V. Avrutin, S. J. Cho, and H. Morkoç, J. Appl. Phys. 98, 041301 (2005).

${ }^{8}$ J. T. Czernuszka and N. Pratt, Philos. Mag. Lett. 61, 83 (1990).

${ }^{9}$ J. E. Bradby, S. O. Kucheyev, J. S. Williams, C. Jagadish, M. V. Swain, P. Munroe, and M. R. Phillips, Appl. Phys. Lett. 80, 4537 (2002).

${ }^{10}$ Z. Takkouk, N. Brihi, K. Guergouri, and Y. Marfaing, Physica B 366, 185 (2005).

${ }^{11}$ M. J. Klopfstein and D. A. Lucca, Appl. Phys. Lett. 87, 131906 (2005).

${ }^{12}$ S. O. Kucheyev, J. E. Bradby, J. S. Williams, C. Jagadish, and M. V. Swain, Appl. Phys. Lett. 80, 956 (2002).

${ }^{13}$ R. Khanna, K. Ip, Y. W. Heo, D. P. Norton, S. J. Pearton, and F. Ren, Appl. Phys. Lett. 85, 3468 (2004).

${ }^{14}$ F. Tuomisto, K. Saarinen, D. C. Look, and G. C. Farlow, Phys. Rev. B 72, 085206 (2005).

${ }^{15}$ F. Leiter, H. Alves, D. Pfisterer, N. G. Romanov, D. M. Hofmann, and B. K. Meyer, Physica B 340-342, 201 (2003).

${ }^{16}$ M. R. Phillips, O. Gelhausen, and E. M. Goldys, Phys. Status Solidi A 201, 229 (2004). 